\title{
Genome sequencing and transcriptome analysis of Geotrichum citri-aurantii on citrus reveal the potential pathogenic- and guazatine resistance- related genes
}

\section{Juan Zhao}

Huazhong Agriculture University College of Horticulture and Forestry Sciences

DeYao Zhang

Huazhong Agriculture University College of Horticulture and Forestry Sciences

\section{Zhe Wang}

Xiangzhou district forestry technology promotion station

\section{Zhonghuan Tian}

Huazhong Agriculture University College of Horticulture and Forestry Sciences

\section{Fan Yang}

Huazhong Agriculture University College of Horticulture and Forestry Sciences

XinJun Lu

Huazhong Agriculture University College of Horticulture and Forestry Sciences

Chao-an Long ( $\square$ postharvest@mail.hzau.edu.cn)

Huazhong Agricultural University https://orcid.org/0000-0002-2028-8553

\section{Research article}

Keywords: Geotrichum citri-aurantii, citrus, genome, pathogenicity, guazatine, drug resistance

Posted Date: December 6th, 2019

DOI: https://doi.org/10.21203/rs.2.18313/v1

License: (9) This work is licensed under a Creative Commons Attribution 4.0 International License.

Read Full License

Version of Record: A version of this preprint was published at Genomics on November 1st, 2020. See the published version at https://doi.org/10.1016/j.ygeno.2020.07.013. 


\section{Abstract}

Background: Citrus grow in more than 100 countries and is one of the most produced fruit genus. Sour rot, caused by Geotrichum citri-aurantii , is a major postharvest disease of citrus \and it causes economic losses. In recent years, the disease had a rising trend year by year. In this study, the genome sequence of G. citri-aurantii and transcriptome sequence of pathogenic- and guazatine resistance were sequenced with a view to explore the potential pathogenic mechanism and drug resistance mechanism of G. citriaurantii on citrus.

Results: We sequenced a high-quality genome sequence of G. citri-aurantii by SMRT. This sequence encodes 6,783 predicted genes of the 28.1-Mb G. citri-aurantii genome. Approximately $5.43 \mathrm{~Gb}$ of clean data were obtained after $\mathrm{Hi}-\mathrm{C}$ sequencing, and a 27.94-Mb genomic sequence was positioned to the 10 chromosome groups after Hi-C assembly , accounting for $99.43 \%$ of the previously measured G. citriaurantii genome. In the process of studying pathogenic mechanisms, the content of polygalacturonase $(P G)$ and polymethylgalacturonase (PMG) was considerably increased in the Newhall navel orange infected by G. citri-aurantii. Then, three polygalacturonase (PG) genes (EVM0005942, EVM0004416, EVM0002276) related to pathogenicity were identified and the expression level was significantly increased during the infection by quantitative RT-PCR. Additionally, G. citri-aurantii is only sensitive to the chemical fungicide guazatine. Massive guazatine use has led to evolution of the wild G. citri-aurantii in citrus-producing areas. Owing to its uniqueness, RNA sequencing analysis of guazatine-resistance showed that the guazatine-resistance of G. citri-aurantii is may related to two ABC transporter family genes, six MFS transporter family genes and two MATE transporter family genes.

Conclusions: We found three polygalacturonase (PG) genes related to pathogenicity and ten genes related to guazatine-resistance from molecular level. Our research may provide novel insights into the effective control of this pathogen.

Keywords: Geotrichum citri-aurantii , citrus, genome, pathogenicity, guazatine, drug resistance

\section{Background}

Sour rot, caused by Geotrichum citri-aurantii (Ferraris), is one of the most important fungal diseases of citrus fruits, affecting all species and cultivars in the world ${ }^{1}$. After being first reported in 1917, this disease has been frequently reported around the world ${ }^{2}$. During the period of harvest, storage, transport and handling, citrus fruits are exposed to the G. citri-aurantii pathogen and can be infected through wounds ${ }^{3}$, especially during rainy seasons.

Despite the severe damage caused by G. citri-aurantii, its infection mechanism and genetic information have not been thoroughly elucidated. Many researchers have tried to control citrus sour rot by physical control, chemical control and biological control ${ }^{4-7}$. However, no suitable methods have been found to 
effectively control the occurrence and spread of sour rot disease in actual production. Thus, obtaining genome information is one of the main strategies to elucidate pathogen infection mechanisms.

Furthermore, guazatine is the only chemical fungicide that can control sour rot effectively ${ }^{8}$. As a broadspectrum bactericidal agent, guazatine plays a role in the biosynthesis of fungal esters, cell membrane function, and inhibiting spore germination ${ }^{9}$, germ tube elongation and adherent cells and hyphae formation. In recent years, the incidence of sour rot has increased in many citrus-producing areas of China. Simultaneously, the use of the guazatine concentration in citrus-producing areas has increased annually. The above-mentioned reports imply that G. citri-aurantii could adapt to the changing environment flexibly. Therefore, the G. citri-aurantii genome may be helpful for understanding the antidrug mechanism of G. citri-aurantii, and it is more conducive to finding an effective method to control sour rot instead of traditional fungicides.

In previous studies, $A B C$, MFS and MATE (multidrug and toxic compound extrusion) transporter family genes play a crucial role in the process of fungal resistance. Two ABC transporter family genes, PMR1 and PMR5, mediate DMI fungicide efflux. The MFS transporter family genes also contributes to fungicide resistance in Penicillium digitatum ${ }^{10}$. The toxic efflux system consisting of PMR1 is directly involved in DMI resistance of fungi ${ }^{11}$. Multidrug efflux transporters play important roles in the regulation of fungal resistance to many DMI drugs. At present, in terms of the primary structure and special energy coupling mode, multidrug efflux transporters are categorized into five families. The MATE family is one of these families ${ }^{12}$.

Sequencing of $G$. citri-aurantii genome and transcriptome open the possibility of identify genes that are related to pathogenic mechanism and guazatine-resistance mechanism. So far, the genome of G. citriaurantii is not recently released.

In this study, we aimed to get which genes in G. citri-aurantii genome associated with pathogenic and guazatine-resistance. Extraordinary, PG could promote infection by some pathogens according to transforming the mechanical strength and rheological properties of the cell wall ${ }^{13}$. ABC transporter family genes, MFS transporter family genes and MATE family genes were proved to evolve resistance to guazatine in P.digitatum genome. For this, we integrated genome sequencing and transcriptome data to study the expression of pathogenic- and drug resistance-related genes.

\section{Results}

\section{The G. citri-aurantii genome sequencing, assembly and annotation.}

The genome of G. citri-aurantii was sequenced by PacBio RS II. A 20-Kb library was constructed for genomic DNA. After filtering the original data, a total of $2.54 \mathrm{~Gb}$ of high-quality sequencing data was obtained (Fig 1A). Assembly led to a genome size of $28.10 \mathrm{Mb}(28,101,572 \mathrm{bp})$ with approximately $90 \mathrm{x}$ 
coverage, divided into 29 scaffolds with a minimum size of $1 \mathrm{Mb}$, as well as an overall $\mathrm{G}+\mathrm{C}$ content of $38.6 \%$ (Table 1). And an overall repeat content of $17.93 \%$ of the genome was discovered through RepeatMasker analysis and PASTEClassifier classification. Some characteristics of G. citri-aurantii genome are shown in Table 1.

6,783 genes were predicted by BRAKER software, with an average length of 2,058 bp, a gene density of 241 genes/Mb and a total of $46.8 \%$ of the genome covered by protein-coding genes. There is an average exon and intron length of $1,203 \mathrm{bp}$ and $111 \mathrm{bp}$.

Ten chromosome groups can be clearly distinguished according to the evaluation of $\mathrm{HI}-\mathrm{C}$ assembly results (Fig. 1C) and displayed by circus plot (Fig. 1D). Within each group, the intensity of the interaction at the diagonal position is higher than the position of the nondiagonal line, and the results indicated that the interaction between adjacent sequences (diagonal positions) is higher in the $\mathrm{Hi}$-C assembled chromosome but not in nonadjacent sequences (nondiagonal position), which is consistent with the principle of $\mathrm{Hi}-\mathrm{C}$ helper genome assembly. After $\mathrm{Hi}-\mathrm{C}$ assembly, the genome sequence length of $27.94 \mathrm{Mb}$ with $193 x$ coverage was mapped to the chromosome, accounting for $99.43 \%$ of the previously measured G. citri-aurantii genome. In the sequence mapped to the chromosome, the sequence length and direction can be determined to be $27.58 \mathrm{Mb}$, accounting for $98.63 \%$ of the total length of the sequence located on the chromosome (Table 1).

In the genome structure, TEs and repetitive DNA sequences play important roles in the evolution of fungi ${ }^{14}$. The total repeat sequences of $5,039,960 \mathrm{bp}(5 \mathrm{M})$ were identified, accounting for $17.93 \%$ of the $G$. citri-aurantii total genome, including LTR retrotransposon, DNA transposon, transposon tandem and other unclassified repeat sequences (Fig. 2A). The number of repeats reached 11,476. Interestingly, Rest of genome are the most repeating sequence (82.17\%). Notably, LINE and LTR account for 5.1 and $2.31 \%$, respectively.

Recently, DNA methylation has also been reported in the class Dothideomycetes ${ }^{15}$. In G. citri-aurantii genome, 171,537 m4C (4-methyl-cytosine) and 11,765 m6A (6-methyl-adenosine) were identified (Fig. 2B). Most of the categorized DNA methylations are m4C. However, m6A DNA methylations occur with high frequency in the regions of repetitive elements compared with m4C (Fig. 2C).

Using the predicted protein sequence of the gene to perform BLAST alignment with functional databases, such as the TCDB database and the PHI database, 170 TCDB and 1,933 PHI were predicted. Then, 196 CAZyme and 1,270 Transmembrane proteins were found through analysis of protein sequences of all predicted genes (Fig. 2D). In G.citri-aurantii genome, The protein containing the transmembrane helix was removed from the predicted 339 proteins containing the signal peptide, and the remaining 158 proteins were secreted proteins. Through domain calling analysis, PF14295.4 $(n=6)$ is the most abundant domain, which mediates protein/protein interactions.

\section{Comparative genomic analysis}


The evolutionary relationship of G. citri-aurantii and other fungi species was analyzed using a group of phylogenetic backbone genes of the fungi. Phylogenetic analysis revealed that $G$. citri-aurantii is evolutionarily close to Galactomyces citri-aurantii, a plant pathogen that has been found on citrus fruit or in the soil of citric fruit orchards (Fig. 3A). In addition, G. citri-aurantii is also close to the other two fungi of Dipodascaceae sp. and Geotrichum candidum (Fig. 3A). To date, in all fungi in the phylogenetic tree of G. citri-aurantii, only the genome of Geotrichum candidum has been published. Synteny analysis of the $G$. citri-aurantii genome and $G$. candidum genome revealed that the $G$. citri-aurantii genome displays different synteny than those fungi (Fig. 3B囚C).

The potential pathogenic related genes analysis of G. citri-aurantii. The plant cell wall is the first barrier of defense against pathogens. Nearly all fungi, particularly necrotrophic pathogens, must initially secrete PGs to dissolve pectin and related components of plant cell wall ${ }^{16-18}$. In vitro, G. citri-aurantii was induced to produce five cell wall-degrading enzymes, polygalacturonase (PG), polymethylgalacturonase (PMG), cellulase (CX), polygalacturonic acid transeliminase (PGTE) and pectinmethyltranseliminase (PMTE) (Fig. 4). PG and PMG have higher activity, and both PGTE and PMTE enzyme activities are less than $1 \mathrm{U} / \mathrm{mg}$ (Fig. 4A). However, in the fruit wounds after culture for $72 \mathrm{~h}$, the activities of only four enzymes, PG, PMG, PGTE and PMTE, were detected. The activity of $C x$ was zero (Fig. 4B). Importantly, the PG activity of the fruit pathological site was significantly higher than that of the healthy part, which is 6.8 times that of the healthy part, and the PMG activity is 1.6 times that of the healthy part.

In G.citri-aurantii genome, three PG enzymes have been discovered, and we identified the genes that produce these three proteins: EVM0005942, EVM0004416 and EVM0002276. The results of quantitative experiments were the same as those of the transcriptome (Fig. 4C). After the citrus was infected by G. citriaurantii, the expression levels of these three enzymes increased greatly, indicating that these three enzymes may play a critical role in the process of $G$. citri-aurantii infection of citrus. 


\section{Transcriptome analysis on the guazatine-}

resistance of $\mathbf{G}$. citri-aurantii.

The guazatine-resistance transcriptome data was repeatable between samples (Fig. 5C). There were $714 \mathrm{DE}$ genes (FDR=2 and FC $=0.05$ ) between AY-1 and AY1-68 of G. citri-aurantii before guazatine treatment and were used as cutoff values (Fig.5E). Among these genes, 385 genes were upregulated, and 329 genes were downregulated. The results indicated that the gene expression pattern was changed significantly in the resistant strains. After guazatine treatment, there were 226 DE genes in AY1-68 that contained 52 downregulated and 174 upregulated DE genes. In addition, $226 \mathrm{DE}$ genes belonging to AY1-68 were uniquely associated with the resistance of AY1-68 to guazatine. There were 108 DE genes between $\mathrm{G} 1$ and $\mathrm{G} 3$ after guazatine treatment, and they were associated with the resistance of AY1-68 to the drug response of guazatine.

All 1146 DE genes and selected genes were classified by Gene ontology (GO) consortium analysis (Fig. 5F). Compared with the wild strain AY-1, the drug-resistant strain AY1-68 could increase its resistance several hundredfold. It is suspected that some of the cellular component organization of this strain has changed to some extent. Simultaneous sequencing revealed that $80 \%$ of these genes have increased expression levels of the transcriptome data. These results revealed that the cellular component of the drug-resistant strain AY1-68 changed.

To identify genes related to the responses of $G$. citri-aurantii to the antifungal drug guazatine and the high resistance of $G$. citri-aurantii, differentially expressed genes of the ABC transporter family and MFS transporter family were further analyzed. The two genes

(EVM0003677, EVM0005437) of the ABC transporter family and six transporter genes (EVM0000087, EVM0000766, EVM0001093, EVM0003101, EVM0004095, EVM0005235) of the MFS transporter 
family received more attention. In this study, RT-qPCR was used to validate the expression levels of eight transporter genes (Fig. 6A, C). these results indicate that overexpression of these genes may enhance the drug resistance of $G$. citri-aurantii.

\section{Transcriptome data analysis revealed that seven MATE transporters (EVM0000063, EVM0002368, EVM0001080, EVM0002368, EVM0003579, EVM0005976, EVM6710) were related to drug} resistance, and these transporter genes were not reported before this study. In the guazatine-resistant strain AY1-68, the expression levels of two MATE genes (EVM0000063, EVM0002368) were upregulated compared with the guazatine-susceptible strain AY-1 (Fig. 6B). The other five MATE genes had decreased expression. Notably, these MATE transporters were normal in AY1-68 after guazatine treatment. These results indicate that two ABC genes and six MFS genes of $G$. citri-aurantii were activated after guazatine treatment. However, two MATE genes may play a decisive role in enhancing drug resistance.

\section{Discussion}

In postharvest diseases of citrus, the incidence of sour rot caused by G. citri-aurantii has increased annually in various producing areas of China ${ }^{9}$. Due to the large-scale use of the fungicide guazatine, the proportion of $G$. citri-aurantiiresistant mutant strains has also increased significantly. In our study, the $G$. citri-aurantii genome of the economically important fungi was sequenced by SMRT for the first time.

The transcripts were analyzed in accordance to the G. citri-aurantii genome. And all the functions in the genome were also identified in the transcriptome. From the total amount of genes, 3 PGs genes were may related to pathogenesis and $\mathbf{1 1 4 6}$ genes were likely to enhance the resistance of sour rot to guazatine. Our preliminary work did not reveal the complex mechanisms of pathogenesis and the guazatine resistance. However, it may lay a foundation for future research on molecular mechanisms.

Epigenetics is currently at the forefront in the identification of pathogen-induced modifications within host plants ${ }^{19}$. However, DNA methylation is an important epigenetic mark associated with plant immunity, but little is known about its roles in fungi infection of citrus ${ }^{20}$. The most studied type of DNA methylation in fungi is m5C, while in prokaryotic cells, it is m4C and m6A. Recently, 171,915 m4C (4methyl-cytosine) and 11,784 m6A (6-methyl-adenosine) (Fig. 2B) in the G. citri-aurantii genome were identified by SMRT for the first time. Surprisingly, large numbers of $\mathrm{m} 4 \mathrm{C}$ were observed in $\mathrm{G}$. citri-aurantii. The $\mathrm{m} 4 \mathrm{C}$ modification occurs with a higher frequency in the region of repetitive elements in the $G$. citri- 
aurantii genome (Fig. 2C), and the results indicate that $\mathrm{m} 4 \mathrm{C}$ may be involved in the transposition of the transposons ${ }^{19}$. Our results also suggest that $\mathrm{m} 4 \mathrm{C}$ may be the focus of future research on sour rot.

PG was first obtained from pectinase produced by pathogenic fungi by Cooper. PG is thought to act by degrading homologous polygalactonic acid regions in plant cell walls ${ }^{21}$. PG is associated with fungal pathogenicity and toxicity by causing tissue degradation and protoplast death ${ }^{22}$. We found three PG genes in the G. citri-aurantii genome, and RT-qPCR results verified that the expression of three PG genes increased. These data further support the hypothesis that PG can promote $G$. citri-aurantii to cause citrus disease.

In drug resistance research of Penicillium digitatum, further research provided evidence that transporter genes from the $A B C$ transporter family and the MFS transporter family have also contributed to fungicide resistance. Two ABC transporter family genes, PMR1 and PMR5, mediate DMI fungicide efflux ${ }^{23}$. MATE (multidrug and toxic compound extrusion) is a multidrug efflux transporter that plays important roles in intrinsic and acquired resistance in many bacteria and in the regulation of fungi resistance to many DMI drugs $^{24}$. In the study of $G$. citri-aurantii resistance, the expression of two genes from the ABC transporter family, six genes from the MFS transporter family and two genes from the MATE transporter family increased in RNA-seq data. These results attracted our attention to study the resistance of G. citri-aurantii to guazatine.

\section{Conclusions}

In summary, we report a complete genome sequence of G. citri-aurantii using the SMRT sequencing method. Then the genome was assembled and annotated. These data not only assist us in finding $17.93 \%$ repetitive elements but also in discovering 171,915 m4C and 11,784 m6A. Using genomic component analysis, gene function annotation and classification, we predicted CAZymes, PHI and TCDB associated with the pathogenicity of sour rot. Additionally, we identified three PG genes related to pathogenesis of $G$. citri-aurantii and some ABC, MFS and MATE genes related to guazatine resistance. Our work also lays the foundation for future research on this important citrus postharvest disease.

\section{Methods}

\section{Fungal strains, culture conditions and DNA extraction.}

A wild-type strain of G. citri-aurantii (AY-1) was isolated from Newhall navel orange in Anyuan city, Jiangxi Province of China. The single-sporing of $G$. citri-aurantii was first obtained by separation and purification by Zhe Wang (2012). Finally, the obtained fungi were formulated into a spore suspension and inoculated onto healthy Newhall navel oranges according to the Koch postulate ${ }^{25}$. The strain is identified as G. citri-aurantii based on the molecular identification of the ITS universal primer and the Blast alignment on the NCBI. 
To obtain the purer mycelia, G. citri-aurantii was cultured on PDA media with a layer of clear cellophane at $28^{\circ} \mathrm{C}$ for $5 \mathrm{~d}$. Genomic DNA of $\mathrm{G}$. citri-aurantii was extracted by using a modified cetyltrimethylammonium bromide (CTAB) method ${ }^{26}$.

\section{Genome sequencing, assembly and chromosomal localization by Hi- C.}

The genome of G. citri-aurantii was sequenced by the single molecule real-time (SMRT) sequencing method with P6-C4 of the latest upgraded chemical reagents. Then, the relationship between the whole chromatin DNA in the whole genome was studied by high-throughput sequencing technology with the Illumina HiSeq platform and Hi-C (high-through chromosome conformation capture) technology, which was combined with the biological information analysis method in Biomarker Technologies (Beijing, China). The sequencing read length is up to PE150.

\section{Genomic component analysis.}

A repetitive sequence library of $G$. citri-aurantii was constructed based on LTR_FINDER, MITE-Hunter, RepeatScout and PILER-DF software. Finally, the repeated sequence prediction of the sequencing data was performed based on the constructed repetitive sequence database using RepeatMasker software.

Gene prediction was performed using de novo prediction, homologous species prediction, and referencebased transcript-based assembly.

An increasing number of studies have found that noncoding RNA plays a significant role in the process of microbial infection. Blastn was used for genome-wide alignment to identify rRNA based on the Rfam database. tRNAscan-SE was used to identify tRNA.

The functional pseudogenes can be recombined by gene conversion into the respective expression site to create a new variant of each gene. Therefore, the study of pseudogenes is particularly important when gene function is studied. Through BLAST alignment, a homologous gene sequence (possible gene) is searched for in the genome. GeneWiseis used to find immature stop codons and frameshift mutations in the gene sequence to obtain pseudogenes.

\section{Genomic function annotation.}

To obtain gene function annotation, the predicted gene sequences were BLAST-matched with functional databases, such as KOG, KEGG, Swiss-Prot, TrEMBL, and Nr. According to the results of the Nr database comparison, the functional annotation of the $\mathrm{GO}$ database is performed using the software Blast2GO.

Protein-encoding genes were annotated by a combination of three databases: The Transporter Classification Database (TCDB), Pathogen Host Interactions (PHI) and CAZyme. 


\section{Whole-genome DNA methylation analysis.}

Based on the results of the de novo assembly, the IPD ratio (the primary metric for kinetic information) was calculated based on the raw data obtained by SMRT sequencing to identify DNA methylation sites ${ }^{27}$.

\section{Synteny analysis and Phylogenetic analysis}

The relevant reference sequences of the ITS sequences of strain AY-1 were downloaded in GenBank using ClustalX 2.0.10 software. Sequence alignment was performed using MUSCLE, and the phylogenetic tree was generated by MEGA 5.0 using a UPGMA method. G. citri-aurantii and Geotrichum candidum were analyzed using GATA.

\section{Extraction and activity determination of the cell wall degrading enzyme of G. citri-aurantii}

Five microliters of the spore suspension $\left(1.0 \times 10^{6}\right.$ spores $\left./ \mathrm{mL}\right)$ of $G$. citri-aurantii was cultured on PDA medium (potato $200 \mathrm{~g}$, sucrose $20 \mathrm{~g}$, agar powder $15 \mathrm{~g}$, distilled water $1 \mathrm{~L}$ ) at $28^{\circ} \mathrm{C}$ for $3 \mathrm{~d}$. The inoculating needle was used to pick the mycelial lump of the colony into the modified Marcus culture medium $\left(\mathrm{KNO}_{3} 2.0 \mathrm{~g}, \mathrm{KCl} 0.5 \mathrm{~g}, \mathrm{FeSO}_{4} 0.01 \mathrm{~g}, \mathrm{~K}_{2} \mathrm{HPO}_{4} 1.0 \mathrm{~g}, \mathrm{MgSO}_{4} \cdot 7 \mathrm{H}_{2} \mathrm{O} 0.5 \mathrm{~g}, \mathrm{VB} 10.1 \mathrm{mg}, \mathrm{L}-\right.$ asparagine $0.5 \mathrm{~g}$, citrus pectin or carboxymethylcellulose sodium salt $10.0 \mathrm{~g}$, distilled water $1 \mathrm{~L}, \mathrm{pH} 5.0$ ) to be grown at $28^{\circ} \mathrm{C}$ and $150 \mathrm{rpm}$ shaking (cellulase culture for $10 \mathrm{~d}$, other enzyme culture for $6 \mathrm{~d}$ ). This step also induces enzyme production.

The hyphae and impurities in the Marcus medium were filtered off by two layers of gauze, and the filtrate was centrifuged twice at $10000 \mathrm{rpm}$ for $15 \mathrm{~min}$; then, the supernatant was combined. Next, $(\mathrm{NH} 4)_{2} \mathrm{SO}_{4}$ was gradually added to the supernatant enzyme solution, slightly vortexed to dissolve, and finally brought to $60 \%$ saturation $\left(25^{\circ} \mathrm{C}\right)$. The mixture was allowed to sit at $4^{\circ} \mathrm{C}$ for $5 \mathrm{~h}$ or overnight. The precipitate was obtained by centrifugation at $12000 \mathrm{rpm}$ for $15 \mathrm{~min}$. Polygalacturonase (PG), polymethylgalacturonase (PMG) and cellulase (CX) were dissolved in $20 \mathrm{~mL}$ of $50 \mathrm{mmol} / \mathrm{L}$ acetic acid-sodium acetate buffer $(\mathrm{pH}=5.0)$ and dialyzed in the buffer. PGTE and PMTE were treated with $20 \mathrm{~mL}$ of $50 \mathrm{mmol} / \mathrm{L}$ glycine$\mathrm{NaOH}$ buffer $(\mathrm{pH}=9.0)$, and the precipitate was dissolved and dialyzed in the buffer. The dialysate was changed once every $12 \mathrm{~h}$ for a total of 3 dialysis cycles, and the enzyme purified by dialysis was stored at $-20^{\circ} \mathrm{C}$.

The spore suspension ( $1.0 \times 106$ spores $/ \mathrm{mL}$ ) of G. citri-aurantii was inoculated into Newhall navel orange and stored for $72 \mathrm{~h}$ at $25^{\circ} \mathrm{C}$ and $95 \% \mathrm{RH}$. The peel sample was taken down with a scalpel around the diseased part of the fruit. In the same way, five cell wall degrading enzymes were extracted and purified.

According to the absorbance value (OD value) of the reducing sugar released by the enzyme reaction, the activity of polygalacturonase (PG), polymethylgalacturonase (PMG), Cellulase (Cx), polygalacturonic acid 
transeliminase (PGTE) and pectinmethyltranseliminase (PMTE) were measured by a UV2450 spectrophotometer in vitro and in vivo. However, in vivo, the sampling time points were set to $36 \mathrm{~h}, 48 \mathrm{~h}$, $60 h, 72 h, 84 h$ and $96 h$.

\section{Transcriptome analysis and quantitative RT-PCR}

For pathogenic transcriptome analysis, $G$. citri-aurantii was cultured on cellophane on PDA medium at $28^{\circ} \mathrm{C}$ for $10 \mathrm{~d}$. The treatment group of $\mathrm{G}$. citri-aurantii was grown in Newhall navel orange at $28^{\circ} \mathrm{C}$ for 10 d. Then, the hyphae and spores were scraped gently using a sterile spoon from the cellophane and the navel orange surface. All samples were frozen at $-80^{\circ} \mathrm{C}$ before transcriptome sequencing. Each treatment had three biological replicates.

The spores and hyphae of $G$. citri-aurantii were used as a control group for drug resistance transcriptome analysis. G. citri-aurantii (AY1-68) is a mutant and is resistant to guazatine with an $\mathrm{EC}_{50}$ value of 6.3793 $\mathrm{mg} / \mathrm{L}$. However, G. citri-aurantii is sensitive to guazatine with an $\mathrm{EC}_{50}$ value of $0.0487 \mathrm{mg} / \mathrm{L}$. G. citriaurantii and the strain AY1-68 were cultured on PDA medium at $28^{\circ} \mathrm{C}$. Then, $20 \mu \mathrm{l}$ of a conidial suspension ( $10^{6}$ spores $\mathrm{ml}-1$ ) of $\mathrm{G}$. citri-aurantii and strain AY1-68 were cultured in $100 \mathrm{ml}$ PDB medium (extract of $200 \mathrm{~g}$ potato boiled water and $20 \mathrm{~g}$ dextrose) at $25^{\circ} \mathrm{C}$ for $72 \mathrm{~h}$, respectively. Then, guazatine $(6.3793 \mathrm{mg} / \mathrm{L})$ was added to PDB with shaking for an additional $6 \mathrm{~h}$ after being cultured at $28^{\circ} \mathrm{C}$ for $48 \mathrm{~h}$. Other groups were added with the same volume of sterile water as a control. The experiment was repeated three times. The samples were as follows: dE6-MI (G. citri-aurantii before the guazatine treatment); PdF6-MI (the strain AY1-68 before the guazatine treatment); PdF6-NI (the strain AY1-68 after the guazatine treatment). The mycelia were filtered and washed several times using double distilled water. All samples were frozen at $-80^{\circ} \mathrm{C}$ before transcriptome sequencing. Each treatment had three biological replicates.

The downloaded data were filtered to obtain clean data, sequence alignment with the specified reference genome ${ }^{28}$, the obtained mapping data, insert length test, randomness test and other library quality evaluation $^{29}$. Structural level analysis of alternative splicing analysis, new gene discovery, and genetic structure optimization were performed on the basis of the clean data. Bioinformatics analysis was performed based on the expression levels of genes in different samples or different sample groups, such as differential expression analysis ${ }^{30}$, functional expression annotation of differentially expressed genes, and functional enrichment.

Total RNA was extracted using RNAiso Plus (TaKaRa Biotech. Co., Wuhan, China) according to the manufacturer's protocol. First-strand cDNA was prepared by NovoScript Plus All-in-one 1st strand cDNA Synthesis SuperMix (gDNA Purge) (Novoprotein, Beijing, China) following the manufacturer's protocol. qRT-PCR was performed using a BIO-RAD CFX96 q-PCR system with SYBR Green I fluorescent dye detection. The mRNA abundance was normalized with the housekeeping gene $\beta$-actin, and the relative expression levels were calculated using the $2-\Delta \Delta C t$ method. 


\section{Declarations}

\section{Ethics approval and consent to participate}

Not applicable

\section{Consent for publication}

Not applicable

\section{Availability of data and materials}

The whole genome sequences and transcriptome data of $G$. citri-aurantii are available at the National Center For Biotechnology Information (NCBI) under PRJNA578961. The genome sequences of $G$. candidum could be found on NCBI (PRJNB5752).

\section{Competing interests}

The authors declare that they have no competing interests.

\section{Funding}

This study was financially supported by National Natural Science Foundation of China (Grant No. 31972122) and the Earmarked Fund for China Agriculture Research System (Grant No. CARS-26).

\section{Authors' contributions}

CAL and JZ planned and designed the research. JZ, YDZ, ZW, ZHT, FY and JXL analysed genomic and transcriptomics data. JZ wrote the manuscript. All authors read, revised and approved the manuscript.

\section{Acknowledgements}

Not applicable

\section{References}

1. Talibi I, Askarne L, Boubaker H, Boudyach EH, Msanda F, Saadi B, et al. Antifungal activity of some Moroccan plants against Geotrichum candidum, the causal agent of postharvest citrus sour rot. Crop Protection. 2012; 35: 41-46. 
2. McKay AH, Forster $H$, Adaskaveg JE. Distinguishing Galactomyces citri-aurantii from G-geotrichum and Characterizing Population Structure of the Two Postharvest Sour Rot Pathogens of Fruit Crops in California. Phytopathology. 2012; 102: 528-538.

3. Klein MN, Kupper KC. Biofilm production by Aureobasidium pullulans improves biocontrol against sour rot in citrus. Food Microbiology. 2018; 69: 1-10.

4. Ferraz LP, da Cunha T, da Silva AC, Kupper KC. Biocontrol ability and putative mode of action of yeasts against geotrichum citri-aurantii in citrus fruit. Microbiological Research. S0944501316301720.

5. Tang X, Ouyang QL, Jing GX, Shao XF, Tao NG. Antifungal mechanism of sodium dehydroacetate against Geotrichum citri-aurantii. World Journal of Microbiology \& Biotechnology 34.

6. Karim H, Boubaker H, Askarne L, Cherifi K, Lakhtar H, Msanda F, et al. Use of Cistus aqueous extracts as botanical fungicides in the control of Citrus sour rot. Microbial Pathogenesis. 2017; 104: 263-267.

7. Liu X, Wang LP, Li YC, Li HY, Yu T, Zheng XD. Antifungal activity of thyme oil against Geotrichum citriaurantii in vitro and in vivo. Journal of Applied Microbiology. 2009; 107: 1450-1456.

8. Zhou YH, Zhang L, Zeng KF. Efficacy of Pichia membranaefaciens combined with chitosan against Colletotrichum gloeosporioides in citrus fruits and possible modes of action. Biological Control. 2016; 96: 39-47.

9. Luo XM, Cao JD, Huang JK, Wang ZY, Guo ZY, Chen YH, et al. Genome sequencing and comparative genomics reveal the potential pathogenic mechanism of Cercospora sojina Hara on soybean. DNA Research. 2018; 25: 25-37.

10. Liu S, Wang WJ, Deng LL, Ming J, Yao SX, Zeng KF. Whole transcriptome analysis of Penicillium digitatum strains treatmented with prochloraz reveals their drug-resistant mechanisms. BMC Genomics. 2015; 16: 855.

11. Hamamoto H, Nawata $O$, Hasegawa $K$, Nakaune R, Lee YJ, Makizumi Y, et al. The role of the ABC transporter gene PMR1 in demethylation inhibitor resistance in Penicillium digitatum. Pesticide Biochemistry and Physiology. 2001; 70: 19-26.

12. Ma QB, Yi R, Li L, Liang ZY, Zeng TT, Zhang Y, et al. GsMATE encoding a multidrug and toxic compound extrusion transporter enhances aluminum tolerance in Arabidopsis thaliana. BMC Plant Biology. 2018; 18.

13. Liu S, Wang WJ, Deng LL, Ming J, Yao SX, Zeng KF. Control of sour rot in citrus fruit by three insect antimicrobial peptides. Postharvest Biology and Technology. 2019; 149: 200-208.

14. Bodega B, Orlando V. Repetitive elements dynamics in cell identity programming, maintenance and disease. Current Opinion in Cell Biology. 2014; 31: 67-73.

15. Ohm R, Goodwin S, Grigoriev I, Dothideomycetes C. Diverse lifestyles and strategies of plant pathogenesis encoded in the genomes of eighteen Dothideomycetes fungi. Phytopathology. 2013; 103: 106-106.

16. Isshiki A, Akimitsu $K$, Yamamoto $M$, Yamamoto $H$. Endopolygalacturonase is essential for citrus black rot caused by Alternaria citri but not brown spot caused by Aternaria alternate. Mol Plant- 
Microbe Interact. 2001; 14: 749-757.

17. Protsenko MA, Buza NL, Krinitsyna AA, Bulantseva EA, Korableva NP. Polygalacturonase inhibiting protein is a structural component of plant cell wall. Biochemistry. 2008; 73: 1053-1062.

18. Zhang L, Kars I, Essenstam B, Liebrand TWH, Wagemakers L, Elberse J, et al. Fungal endopolygalacturonases are recognized as microbe-associated molecular patterns by the arabidopsis receptor-like protein responsiveness to botrytis polygalacturonases1. Plant Physiology. 2014; 164: 352-364.

19. Dubey A, Jeon J. Epigenetic regulation of development and pathogenesis in fungal plant pathogens. Molecular Plant Pathology. 2017; 18: 887-898.

20. Sun Y, Fan M, He Y. DNA Methylation Analysis of the Citrullus lanatus Response to Cucumber Green Mottle Mosaic Virus Infection by Whole-Genome Bisulfite Sequencing. Genes. 2019; 10: 5.

21. Liu N, Li HJ, Chevrette MG, Zhang L, Cao L, Zhou HK, et al. Functional metagenomics reveals abundant polysaccharide-degrading gene clusters and cellobiose utilization pathways within gut microbiota of a wood-feeding higher termite. Isme Journal. 2019; 13: 104-117.

22. Carrasco M, Rozas JM, Alcaino J, Cifuentes V, Baeza M. Pectinase secreted by psychrotolerant fungi: identification, molecular characterization and heterologous expression of a cold-active polygalacturonase from tetracladium sp. Microbial Cell Factories. 2019; $18: 1$.

23. Marcet-Houben M, Ballester AR, de la Fuente B, Harries E, Marcos JF, Gonzalez-Candelas L, et al. Genome sequence of the necrotrophic fungus penicillium digitatum, the main postharvest pathogen of citrus. BMC Genomics. 2012; 13: 646-646.

24. Morita Y, Kodama K, Shiota S, Mine T, Kataoka A, Mizushima T et al. NorM, a putative multidrug efflux protein, of Vibrio parahaemolyticus and its homolog in Escherichia coli. Antimicrobial Agents and Chemotherapy. 1998; 42: 1778-1782.

25. Wang J, Fedoseienko A, Chen BY, Burstein E, Jia D, Billadeau DD. Endosomal receptor trafficking: retromer and beyond. Traffic. 2018; 19: 578-590.

26. Chin CS, Alexander DH, Marks P, Klammer AA, Drake J, Heiner C, et al. Nonhybrid, finished microbial genome assemblies from long-read SMRT sequencing data. Nature Methods. 2013; 10: 563-.

27. Flusberg BA, Webster DR, Lee JH, Travers KJ, Olivares EC, Clark TA, et al. Direct detection of DNA methylation during single-molecule, real-time sequencing. Nature Methods. 2010; 7: 461-U472.

28. Kim D, Landmead B, Salzberg SL. Hisat: a fast spliced aligner with low memory requirements. Nature Methods. 2015; 12: 357-360.

29. Pertea M, Pertea GM, Antonescu CM, Chang TC, Mendell JT, Salzberg SL. StringTie enables improved reconstruction of a transcriptome from RNA-seq reads. Nature Biotechnology. 2015; 33: 290-t.

30. Florea $L$, Song $L$, Salzberg SL. Thousands of exon skipping events differentiate among splicing patterns in sixteen human tissues. F1000Research. 2013; 2: 188.

\section{Tables}


Due to technical limitations, Table 1 is only available as a download in the supplemental files section.

\section{Figures}
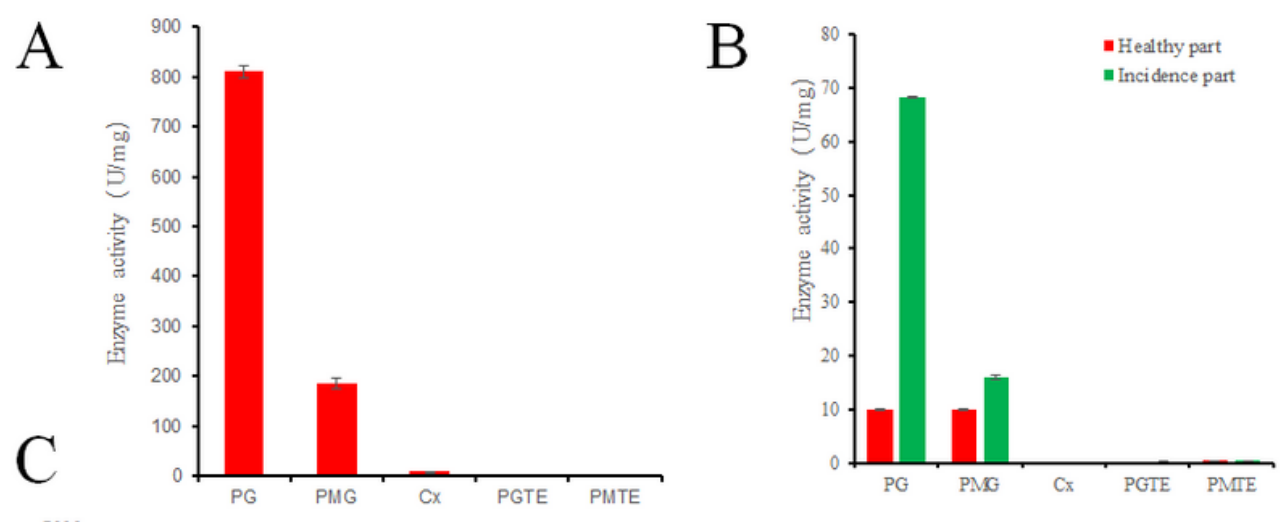

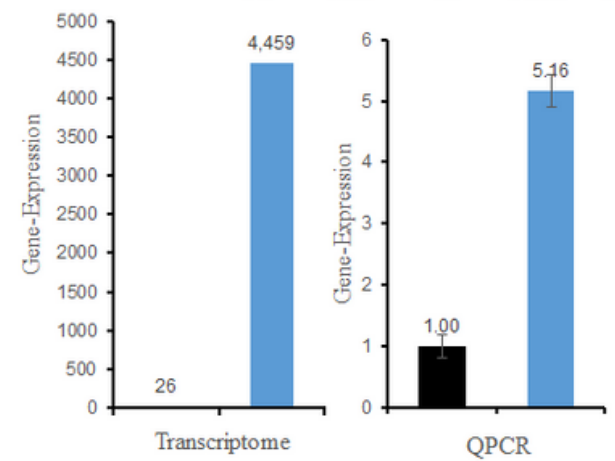

EVM0005942

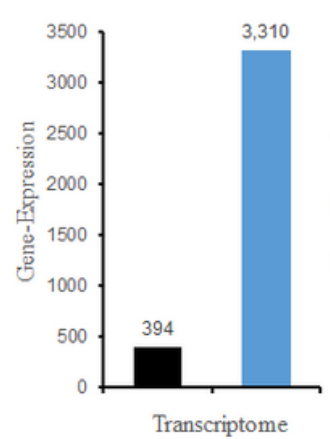

EVM0004416

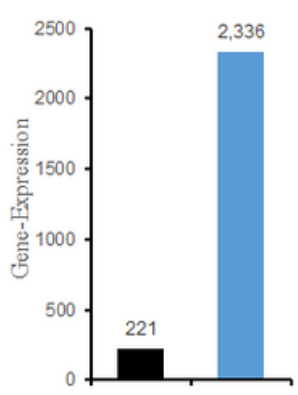

Transcriptome

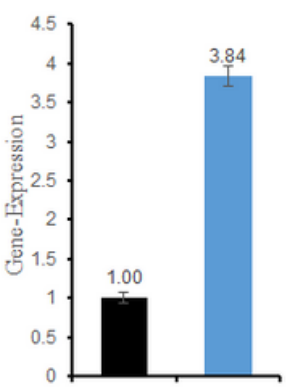

QPCR

EVM0002276

Figure 1

Determination of cell wall-degrading enzyme activity of $G$. citri-aurantii and expression analysis of three PG genes. A. Activity of cell wall degrading enzymes produced by G. citri-aurantii in vitro; B. Activity of cell wall degrading enzymes produced by incidence and healthy part; $C$. Expression analysis of 3 PG genes after infection with citrus. The mRNA abundance was normalized using the housekeeping gene actin, and the relative expression levels were calculated using the $2-\Delta \Delta C t$ method. Three biological replicates were performed. 
A
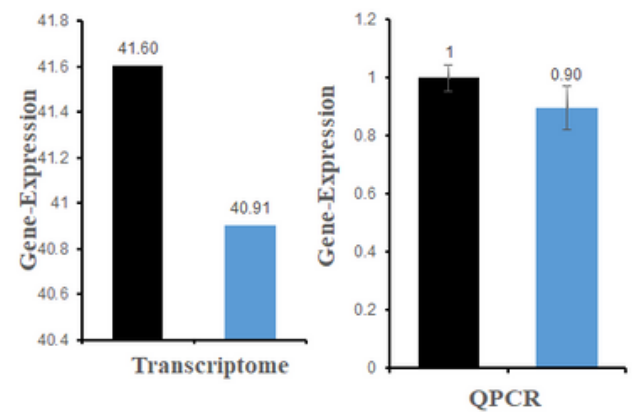

EVM0003677

B
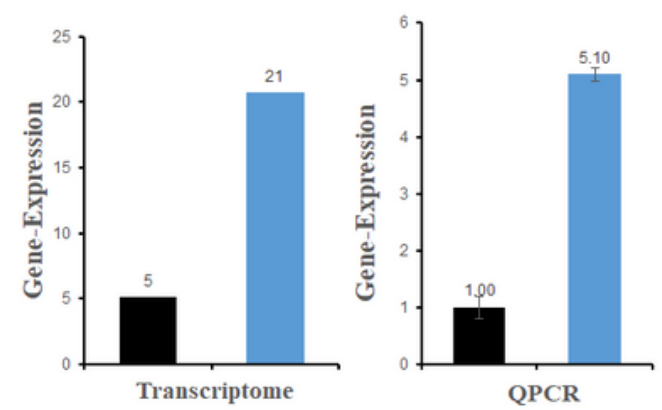

EVM0000063

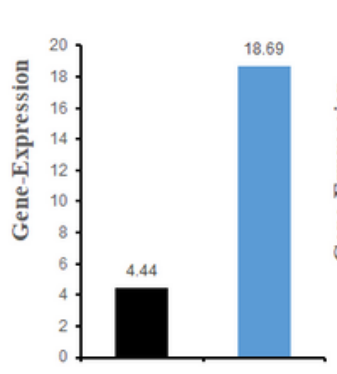

Transcriptome

EVM000543

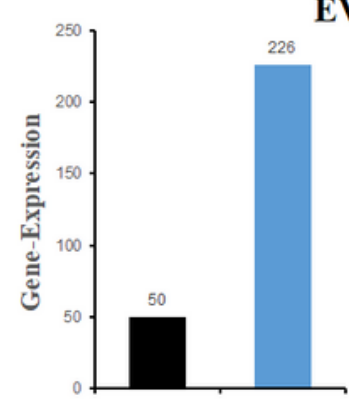

Transcriptome

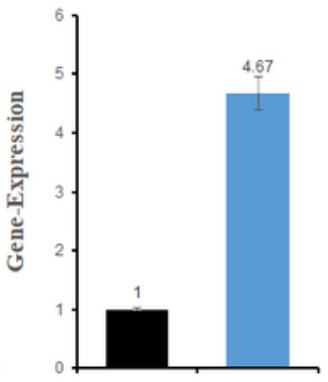

QPCR

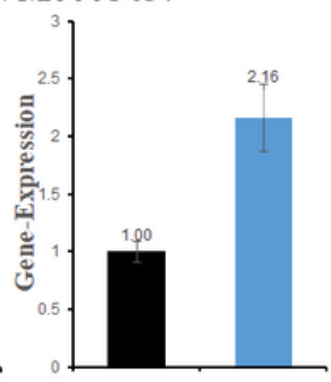

EVM0002368

C

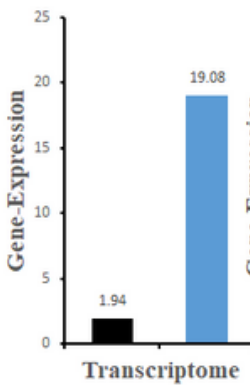

EVM0000087

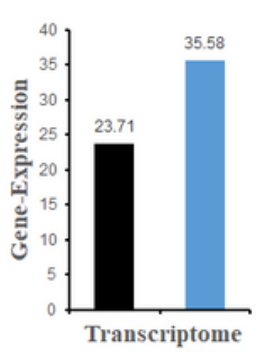

EVM0003101

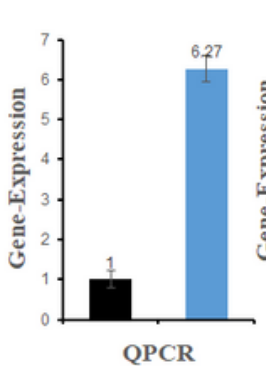

QPCR

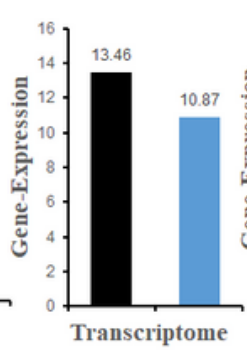

EVM0000766
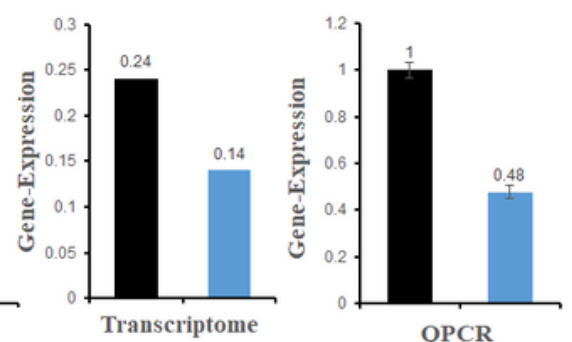

EVM0001093
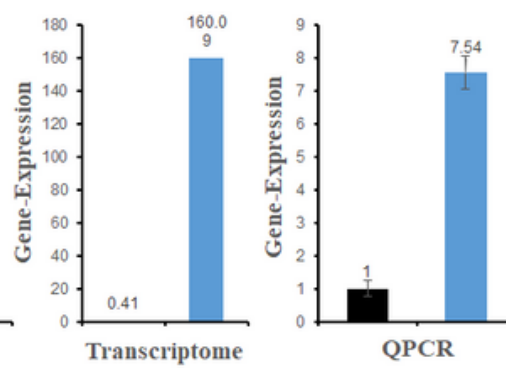

EVM0004095
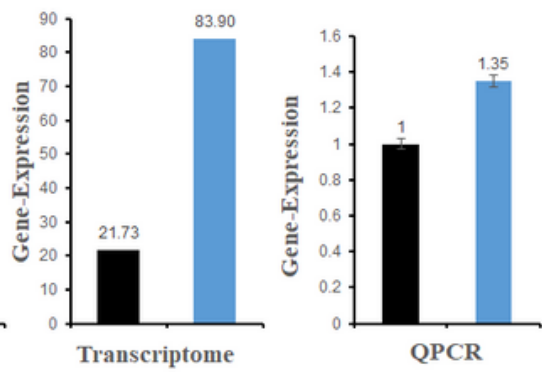

EVM0005235

Figure 2

Expression analysis of chosen genes of the ABC family, MFS family and MATE family genes. A. Expression analysis of chosen transporter genes of ABC family genes in AY1-68 after albesilate treatment; B. Expression analysis of chosen transporters of MATE family genes in AY-1 and AY1-68; C. Expression analysis of chosen transporter genes of MFS family genes in AY1-68 after albesilate 
treatment The mRNA abundance was normalized using the 18s housekeeping gene, and the relative expression levels were calculated using the $2-\Delta \Delta C t$ method. Three biological replicates were performed.

\section{Supplementary Files}

This is a list of supplementary files associated with this preprint. Click to download.

- Table1.png 